\title{
Sentence encoding and implicitly activated memories
}

\author{
DOUGLAS L. NELSON, NANCY R. GEE, and THOMAS A. SCHREIBER \\ University of South Florido, Tampa, Florida
}

\begin{abstract}
Some words have fewer direct associates than others, and, when words varying in set size are studied in a list-learning task, those with smaller sets are more likely to be recalled. This setsize effect is found in cued recall when the words are studied in the absence of related words, but not when studied in the presence of related words. Related words provide context and theoretically inhibit irrelevant associates. The present research determined that set-size effects are found when words are encoded in sentence contexts. In contrast to list-learning experiments, the results of three experiments found such effects even when lexically related words were present in the sentences. Other findings indicated that target-set-size effects were determined by the proximity of related words in the sentence and the nature of the test cue. The results are discussed in relation to a model for explaining set-size effects and to selective findings from the sentencecomprehension literature.
\end{abstract}

English words are associatively connected to varying numbers of other words, and a great deal of research has been devoted to understanding the role of such connections in the comprehension and recall of word lists and sentences (e.g., Kintsch, 1988; Nelson, Schreiber, \& McEvoy, 1992). The general purpose of the present experiments was to determine whether the number of associates linked to a word presented in the context of a sentence would influence its recall. The research is based on a consistent finding obtained in list-learning experiments: when recall is prompted by meaningfully related test cues, recently studied words linked to smaller numbers of associates are more likely to be recalled than are those that are linked to larger numbers of associates. The associative set size of a studied word affects the likelihood of its recall, and this phenomenon is called the targetset-size effect (e.g., Nelson \& Friedrich, 1980). This effect is apparent for concrete and abstract words (Nelson \& Schreiber, 1992) and occurs regardless of whether subjects encode the words by using intentional learning strategies, by rating them for concreteness or pleasantness, or by counting vowels (Nelson, Bajo, \& Casanueva, 1985; Nelson, Schreiber, \& McEvoy, 1992). The set-size effect occurs at both fast and slow presentation rates (Nelson, Schreiber, \& McEvoy, 1992) and is maintained after studying up to four lists of either related or unrelated words prior to the cued-recall test (Nelson, McEvoy, Janczura, \& Xu, 1992).

This research was supported by Grant MH 16360 from the National Institute of Mental Health to the first author. Our special thanks go to Shirley Peffers, Candice Stewart, and Page Moore for helping with the data collection and to Harry Bahrick and two anonymous reviewers for their helpful comments on an earlier draft of the paper. Correspondence concerning this article should be sent to D. L. Nelson, Department of Psychology, University of South Florida, Tampa, FL 33620.
These findings indicate that the target-set-size effect is a robust phenomenon, but other findings indicate that the effect is highly sensitive to variables affecting the likelihood of searching through associates of the target during testing. First, the effect depends on the nature of the information represented in the test cue. Target-set-size effects are apparent in cued-recall tasks (Nelson, Schreiber, \& McEvoy, 1992), but they are not obtained in either fragment-completion tasks (Nelson, Keelean, \& Negrao, 1989) or recognition tasks where the targets serve as test cues (Nelson, Canas, \& Bajo, 1987). Second, although studying additional lists of words does not alter the magnitude of the effect, asking subjects to do multiplication problems for a comparable period eliminates it (Nelson, McEvoy, Janczura, \& Xu, 1992). Third, presenting the target in the presence of a directly associated word during study reduces and often eliminates the target-set-size effect (e.g., Nelson \& Friedrich, 1980; Nelson, McEvoy, \& Schreiber, 1990). In general, certain types of test cues and attention-switching procedures, and, most important, the presence of related words all decrease the likelihood of finding target-set-size effects. These variables decrease the probability of searching through associates of the target during testing.

The sensitivity of the target-set-size effect to the nature of the test cue, to attentional demands, and to the presence of meaningful context raises questions about the generality of the effect. The phenomenon may occur only under circumscribed conditions, and, although it has served as a useful tool for investigating the function of preexisting connections, the phenomenon may hold little value for exploring other issues. For example, the semantic constraints created in the process of comprehending even simple sentences such as "John watched the hive" may eliminate target-set-size effects. If such effects are eliminated in simple sentential contexts, set-size manipu- 
lations would be useless as a tool for investigating processes involved in the recall of sentence material. Alternatively, the presence of these effects in sentences would have implications for comprehension processes and could serve as a means for determining when preexisting knowledge is being utilized in sentence processing or discoursecomprehension tasks (e.g., Kintsch, 1988). These considerations motivated the present experiments, which were designed to determine whether set-size effects occur when target words are presented in the context provided by a sentence. The presence of set-size effects would indicate that associates of the target were activated during sentencecomprehension and encoding processes and that the reactivation of these associates during testing can support subsequent recall.

In Experiments 1 and 2, subjects studied sentences in which the target word always appeared as the last word in each sentence. As in the list-learning experiments, half of the targets had small sets and half had large sets of associates as determined by prior measurement, and the meaningfully related word used as the test cue was either present or absent in the sentence. When the test cue was absent, subjects studied sentences like "John watched the hive"; when the test cue was present, each sentence included the word used as the test cue, but the cue and target were often separated by other words, as in "John watched the bee fly around the hive." In both conditions, recall of the last word in the sentence was cued by a meaningfully related word (e.g., the word "bee"). The conditions of encoding and retrieval were similar to those typically used in the list-learning experiments, but the critical words were not underlined or isolated in any way from the rest of the sentence. Recall was assessed using both accuracy and response time in Experiment 1. Experiment 2 directly compared meaningfully related cues such as "bee" and sentence-frame cues such as "John watched the __ " or "John watched the bee fly around the ." Interest in both experiments focused on the magnitude of target-set-size effects as a function of the presence of a related word in the sentence and the type of test cue, word or sentence frame. Experiments $3 \mathrm{~A}$ and $3 \mathrm{~B}$ were designed to determine whether the proximity of meaningfully related words is critical to reducing the magnitude of observed target-set-size effects in both list- and sentence-learning tasks.

Predictions concerning target-set-size effects in the present experiments depend upon what assumptions are made about the role of the sentence context in restricting the activation of related associates. Our initial expectations were based on a model designed to explain target-set-size effects in list-learning experiments (Nelson, Schreiber, \& McEvoy, 1992). The model, called "PIER," assumes that recall is determined by processing implicit and explicit representations. The model assumes that success in cued recall is determined by searching explicit memories created as a result of controlled processing activities (e.g., Raaijmakers \& Shiffrin, 1981) or by searching implicit memories activated during the task (Nelson, 1989). During the study phase, intentional encoding operations applied to the stimulus materials produce an explicit representation, and a second representation is established independently of the nature of these operations. This second, implicit representation consists of associates of the words being processed, which are automatically and implicitly activated as an unavoidable consequence of comprehension.

The presence of context provided by meaningfully related words determines which of the activated associates become a functional part of the implicit representation. When meaningfully related context words are absent, close associates of the target are activated and incorporated into the representation. In contrast, when the target is presented in the context of a meaningfully related word, only associates shared by the context word and target are included in the representation. Irrelevant associates are activated initially but then inhibited, and, as a result, the functional set size of targets with large associative sets approaches the functional set size of those with small sets.

In PIER, the probability of a successful explicit search depends on how well the target itself has been encoded in relation to the context and to the cue used to prompt its recall. The probability of a successful implicit search depends on the nature of the information represented in the test cue and on the number of activated associates. Target-set-size effects occur when related context words are absent during study because directly related associates have been activated. The probability of sampling the target from among its competing associates is greater and the process takes less time when the activated set is small compared with when the set is large. In this model, the activation of associates determines the pool of items that compete for the target, and set-size effects arise from the retrieval inhibition encountered in sampling associates in this pool. Target-set-size effects are reduced when related context cues are present during study because fewer associates are incorporated into the implicit representation and because there are fewer associates competing with the target during recall.

According to PIER, target-set-size effects are contingent on the context in which the target appears. This rationale suggests that target-set-size effects should be evident when words are encoded in a sentence context whenever that context fails to constrain the meaning of the target. In the present experiments, such effects should be evident when the related context words are absent because none of the words in these sentences are directly associated to the target; for example, neither "John" nor "watched" is directly associated to the target word "hive." Target-set-size effects may or may not be evident when related context words are available in the sentence. The presence of such effects should depend on whether the context constrains functional activation to the same degree as a directly related word in a list-learning experiment where related word and target are presented simultaneously. In normal sentences, directly related words are often separated by lexically unrelated words. To the extent that the inhibition of associates depends on 
the timing of the related word experience, target-set-size effects that normally are reduced and sometimes are eliminated in list-learning experiments should be present in sentence-learning tasks.

Similar expectations for the present experiments were based on the findings available in the sentencecomprehension literature. Many studies have demonstrated that a sentential context can influence the nature of the representation of a word that is constructed (e.g., Gernsbacher, Varner, \& Faust, 1990; Seidenberg, Tanenhaus, Leiman, \& Bienkowski, 1982; Tabossi, 1988a, 1988b). Tabossi (1988a) showed that lexical access can be affected by prior sentential context, but only when that context imposed semantic constraints on words appearing later in the sentence. Subjects listened to a sentence containing an ambiguous word (e.g., "port") and performed a lexical decision task on a visually presented control word or on a word related to one of its meanings. The sentence biased the dominant meaning of the ambiguous word and either did or did not place a semantic constraint on it (e.g., "The violent hurricane did not damage the ships which were in the port, one of the best equipped along the coast" should be compared with "The man had an appointment at the port at five o'clock"'). Relative to control words, subjects responded faster to words related to both meanings in the unconstrained context sentence (e.g., "safe," "red"), but they responded faster only to the primed meaning (e.g., "safe") in the constrained context. The presence of related words in the sentence (e.g. "ships") apparently constrained or inhibited the activation of words related to the unprimed meaning. Similar findings have been reported by Seidenberg et al. (1982). These authors used a naming task and showed that both meanings of an ambiguous word were activated when the sentential context did not contain words that were lexically related to the ambiguous word, whereas only a single meaning was activated when the sentence contained such words. The results presented in these two papers suggest that the activation of associatively related words is constrained by sentential contexts, but only when meaningfully related words are present in the sentence. Seidenberg et al. suggest that differences between these findings and those of Onifer and Swinney (1981) were likely to be due either to the absence of related words in their sentences or to the fact that, when present, "the related words occurred four or more words before the ambiguous word" (Seidenberg et al., 1982, p. 528).

These results indicate that the presence of related words in sentence contexts can influence the activation process. These findings, like PIER, provide a basis for the expectation that the magnitude of target-set-size effects will vary with the presence of meaningfully related words in the sentential context. Target-set-size effects should be evident in sentences where related words are absent, and they should be reduced when related words are present, but the amount of reduction should depend on the proximity of the related words in the sentence.

\section{EXPERIMENT 1}

\section{Method}

Design. The experimental design formed a $2 \times 2$ mixed-model factorial. Presence of a directly related word in the sentence (present, absent) was the between-subject variable, and target-set size (small, large) was the within-subject variable.

Subjects. Sixteen different subjects served in each between-subject condition, with 8 assigned to each sentence list. The subjects were drawn from courses in introductory psychology, were awarded points for their participation, and were assigned to conditions in replication blocks.

Materials. The targets and their meaningfully related test cues for the two lists of sentences used in this experiment were taken from a previous list-learning study and can be found in that repor (Nelson et al., 1990, Experiment 2). The original lists were constructed with the use of a normative database of approximately 2,000 words. Many groups of subjects $(n=100-200)$ were given approximately 100 different words and were asked to write the first word that came to mind that was meaningfully related to or that was strongly associated with each provided word. Their responses were used to estimate both strength and associative set size. The probability of any given associate was used to estimate the strength of the preexisting relationship between the normed word and any particular response, and the number of different associates given by 2 or more subjects was used to estimate associative set size for the normed word (see Nelson \& Schreiber, 1992, for additional details and rationale).

The sentences are presented in Appendix A. In the contextword-absent condition, simple sentences were constructed around these words such as "John saw the calf," "Mary asked for a clock," and so forth. The first word in each sentence always named a different person, each sentence used a different verb, and the target word always appeared as the last word in the sentence. In the contextword-present condition, the related context word appeared 0-4 words before the target and was placed to maintain the flow of the sentence (e.g., "John saw the cow produce the calf," "Mary needed to know the time and asked for a clock"). In each of these conditions, half of the targets had small sets of associates $(7.13, S D=1.09)$ and half had large sets $(19.34, S D=2.38)$. None of the words in the sentences was underlined. The stems used for the small targets in Sentence Set 1 were used for the large targets in Sentence Set 2, and vice versa, with only minimal changes in wording to preserve meaning. For example, the stem "John watched the ..." was used for the small-set-size word "hive" in Sentence Set 1 and for the large-set-size word "wine" in Sentence Set 2.

The sentences in the context-word-absent condition were shorter ( 4.38 words) than those in the context-word-present condition ( 7.60 words). This confounding was intentional, as we wanted to determine whether target-set-size effects would be apparent in the simplest sentences that we could write for the absent condition, and the present condition required additional words to maintain the sentence flow. The confounding was not regarded as a serious problem, because our intent was to evaluate the relative magnitude of target-set-size effects within each of these conditions. Furthermore, although the memory load was expected to be greater for the longer sentences, our list-learning studies have indicated that recall is considerably better when the context word is present during study (Nelson, Schreiber, \& McEvoy, 1992).

The words used as the test cues (e.g., for the above sentences, "cow" and "time') were selected from the associative norms to produce the targets with an average probability of $.23(S D=.10)$. Cue-set size averaged 12.79 related associates $(S D=3.84)$. Both cue-to-target strength and cue-set-size affect the probability of cued recall, and both variables were carefully equated at each level of target-set size in order to ensure that the target-set-size manipula- 
tion was not confounded with characteristics of the test cue (e.g., Bahrick, 1970; Nelson \& McEvoy, 1979).

Procedure. The procedures used for presenting and testing the recall of the sentences were similar to those used for previous listlearning experiments, except that the study trial was paced at a 12sec rate instead of a 4-sec rate (Nelson et al., 1990). Each of the 24 sentences was displayed one at a time on an Apple computer screen, and the subjects were asked to read each sentence aloud as it appeared and to remember as many as possible. They were told that each sentence would be presented only once and that they would be asked some questions about the sentences later. The ordering of all sentences was random and was changed between subjects. Before the presentation of the sentences, the subjects were shown six word pairs and were asked to remember them. Immediately afterwards, the leftmost word was shown as the cue to recall the related right-hand word by speaking it aloud. The purpose of this task was to acquaint the subjects with the rate of presentation, using the computer, and the cuing task.

Immediately following the last sentence, the instructions for the test trial were read to the subject. These instructions indicated that each cue was meaningfully related to the last word of one of the sentences. The subjects were told to respond as quickly and as accurately as possible but to guess when unsure. Each response was timed from the onset of the cue to the onset of the response by using a voice-key and digital timer. The orders of the cues were independently randomized for each subject.

\section{Results and Discussion}

Table 1 shows probabilities of correct recall and response latency calculated for correct recall as a function of presence of the related word in the study sentence and target-set size. Comparable results for accuracy from the list-learning experiment based on the same words are also presented in the table for comparison. These results were part of a larger experiment concerning target-set-size effects in both cued- and free-recall tasks (see Nelson et al., 1990, Experiment 2).

As in the list-learning experiment, the findings of the sentence experiment indicated that targets with smaller associative sets were more likely to be recalled than those with larger sets. Recall was also more likely when the meaningfully related test cues were present during study, and the presence of these cues tended to reduce somewhat the magnitude of the target-set-size effect. However, when the cues were present during study, the target-setsize effect was more apparent in the sentence experiment than in the list-learning experiment. The statistical analysis of the list-learning experiment indicated that cue presence $\left[F(1,30)=23.20, M S_{\mathrm{e}}=.054\right]$, target-set size $\left[F(1,30)=37.32, M S_{\mathrm{e}}=.007\right]$, and the interaction of these sources $[F(1,30)=18.73]$ were all significant sources of variance. In contrast, in the sentence experiment, the effects of cue presence $[F(1,30)=16.87$, $\left.M S_{\mathrm{e}}=.04\right]$ and target-set size $\left[F(1,30)=19.25, M S_{\mathrm{e}}=\right.$ $.01]$ were significant, but the interaction between these sources was not $(F=2.31, p<.14)$.

In the sentence experiment, similar effects were also apparent for the latency measure. Response latencies were faster in the cue-present condition and for targets with smaller sets. Effects of cue presence $[F(1,30)=5.02$, $\left.M S_{\mathrm{e}}=26.62\right]$ and target-set size $[F(1,30)=5.89$, $\left.M S_{\mathrm{e}}=3.98\right]$ were reliable, but their interaction was not $(F<1)$. On average, the subjects were $2.89 \mathrm{sec}$ faster when the test cue was present during study than when it was absent and $1.21 \mathrm{sec}$ faster when the targets had small sets than when they had large sets.

The present findings indicated that target-set-size effects were apparent when the critical words were studied in the context of simple sentences. Compared with words with large associative sets, words with smaller sets were more likely to be recovered and took less time to be recovered. These findings indicate that such effects can be found outside of the list-learning paradigm. However, targetset-size effects were not significantly reduced when the word serving as the test cue was also presented as part of the sentence. This finding contrasts with the listlearning experiments and is presumably related to the separation of the preexperimentally related words under the sentence-encoding condition. In the list-learning experiment, the related items were directly paired, whereas in the sentence experiments, they were often separated by intervening words to maintain the flow of the sentence. However, before considering the implications of these findings any further, the results of Experiment 2 will be described. This experiment was designed as a replication of the sentence experiment, but recall was tested using meaningfully related cues for one group of subjects and sentence-frame cues for another group of subjects.

\section{EXPERIMENT 2}

One purpose of Experiment 2 was to replicate Experiment 1 using meaning cues and a new sample of subjects, and another purpose was to prompt recall of the target using sentence frames as test cues. The findings were expected to vary with the presence of related words in the sentence, and different results were expected for the two types of test cues: When related words were present during study, the typical set-size pattern was expected for

Table 1

Probability of Correct Recall and Latency (in Seconds) as a Function of the Presence of a Context Word/Test Cue During Study and of Target-Set Size

\begin{tabular}{lccccc}
\hline & \multicolumn{2}{c}{ Test Word Present at Study } & \multicolumn{2}{c}{ Test Word Absent at Study } \\
\cline { 2 - 3 } \cline { 5 - 6 } & Small Target & Large Target & & Small Target & Large Target \\
\hline List task (Nelson et al., 1990) & .84 & .81 & .64 & .44 \\
Sentence task (accuracy) & .77 & .69 & & .61 & .44 \\
Sentence task (latency) & 3.03 & 4.12 & & 5.80 & 7.13 \\
\hline
\end{tabular}


the word cues but not for the sentence-frame cues. Finding set-size effects for the word cues would replicate the results of Experiment 1 and would suggest that some of the associates of the target were not effectively inhibited by the prior appearance of the related context word in the sentence. In contrast, the presentation of the sentence frames as test cues should eliminate the set-size effect altogether. Such cues include all the words of the sentence except the last and they incorporate constraints produced by both related words and the sentence context as a whole (e.g., "John watched the bee fly around the "). As a consequence, recall should be based primarily if not exclusively on search of the explicit representation, and typical target-set-size effects should not be found.

When the related words were absent in the sentence, we expected to find the usual pattern when meaningfully related cues were used to prompt recall. As in Experiment 1 , targets with smaller associative sets should have a greater likelihood of being recalled. However, for frame cues, reversed set-size effects were expected. Although reversed effects are typically small, previous findings using lists have shown that targets with larger sets of associates are more likely to be recalled than are those with smaller sets when encoded in the presence of unrelated words used later as recall cues (Nelson et al., 1990). Given unrelated word pairs, the probability of activating remote connections between them should be greater when many associates are activated than when only a few associates are activated. PIER assumes that the activation of remote connections between a context word and the target mediates and facilitates learning the cue-target relationship (also see Underwood \& Schulz, 1960). The same principle should also hold for sentences when none of the words are related to the target. Hence, when related words are absent in the sentences during study, different results are predicted for frame and meaning cues. For frame cues, recall should be more probable when set size is large, and for meaningfully related word cues, recall should be more probable when set size is small.

\section{Method}

Design and Subjects. The experimental design formed a $2 \times$ $2 \times 2$ factorial. Presence of a related word in the sentence (present, absent) and type of test cue (meaning, frame) were manipulated between subjects, and target-set size (small, large) was varied within subjects. Sixteen subjects served in each between-subject condition.

Materials and Procedure. The targets, test cues, and sentences for the two lists were identical to those used in Experiment 1. The study and test procedures were also identical to those used in the initial experiment, but the materials were presented on a Macintosh Plus rather than an Apple computer and latencies were not recorded.

\section{Results and Discussion}

The principal findings are presented in Table 2 . As can be seen, the findings conformed to expectations for the manipulation of the presence of related words in the sentences. When related words were present, small targetset-size effects were apparent when recall was cued by meaningfully related words, as in Experiment 1 . Such effects, however, were eliminated when recall was cued by the sentence-frame cues. Recall was relatively high and was as likely for words with large associative sets as it was for words with small associative sets.

By contrast, when related words were unavailable during study, set-size differences were evident for both meaningfully related cues and for sentence-frame cues. These differences, however, were related in a crossover interaction. When prompted by meaning cues, recall was higher for words with smaller sets, and when prompted by sentence-frame cues, recall was higher for words with larger sets.

These observations were supported by the results of the statistical analysis, which showed that, as in Experiment 1 , the effects of cue presence $[F(1,60)=77.08$, $\left.M S_{\mathrm{e}}=.05\right]$ and target-set size $\left[F(1,60)=3.68, M S_{\mathrm{e}}=\right.$ $.01]$ were significant, but the interaction between these sources was not $(F<1)$. Type of test cue showed no main effect $(F<1)$ but interacted significantly with cue presence $[F(1,60)=18.97]$, with target-set size $[F(1,60)=12.82]$, and with both of these variables $[F(1,60)=4.19]$. Fisher's two-tailed least significant difference $(L S D)$ for the reliable three-way interaction was .07 . This interaction reflected the differing effects of set size across the conditions of cue presence and type of cue.

The results of Experiments 1 and 2 indicated that when recall was prompted by meaningfully related cues, targetset-size effects were apparent when the related cues were absent in the sentence. The sentential constraint provided by the sentences used in these conditions was insufficient to constrain the activation of the closest associates of the targets. In other words, when subjects studied such sentences as "John watched the hive" and "Tom held the baby," close associates of the targets "hive" and "baby" were functionally activated and played an important role when recall was prompted by meaningfully related cues.

Close associates also played an important role in that they facilitated the making of connections among unrelated words in the sentence, as shown by the reversed set-size effects obtained with sentence-frame cues such as "John watched the " and "Tom held the

Theoretically, the activation of associates of the target provides access to more remote associates that can provide

Table 2

Probability of Correct Recall as a Function of the Presence of a Context Word During Study, of Target-Set Size, and of Type of Test Cue

\begin{tabular}{cccccc}
\hline & \multicolumn{2}{c}{ Test Cue Present at Study } & & \multicolumn{2}{c}{ Test Cue Absent at Study } \\
\cline { 2 - 3 } \cline { 5 - 6 } Type of Test Cue & Small Target & Large Target & & Small Target & Large Target \\
\cline { 2 - 3 } Word & .80 & .74 & & .68 & .63 \\
Frame & .90 & .90 & & .36 & .43 \\
\hline
\end{tabular}


mediating links between the target and other words in the sentence, and the more direct associates there are, the greater the chances of producing an effective mediating link. However, the effects of these mediating links are only likely to be apparent when recall is cued with the original unrelated context words such as "John watched" because the links are between these words and the remote associates. The reverse target-set-size effect is the product of context-dependent encoding and will be apparent only when the original context is reinstated at test.

Although to a slightly lesser extent, the typical targetset-size pattern showing higher recall for words with smaller sets was also apparent when related words were present in the sentence during encoding and used as test cues. As in Experiment 1, presenting associatively related words in the sentences did not eliminate the influence of closely connected associative information. However, when such words were present and the test cues consisted of the sentence frames that included these words, set-size effects were eliminated. This finding suggests that the combined constraints provided by other words in the sentence besides the related word can contribute to the elimination of the target-set-size effect, but that these other words must be present during testing to reexert their constraining effects. The source of this constraint appears to be context dependent and may involve the activation of remote associates of the target rather than the activation of its more context-independent associates (Barsalou, 1982). For example, the sentence "John watched the bee fly around the hive" may activate a context-dependent "home" meaning of hive implying that the bee is flying around its home, but this constraint may be incapable of restricting the breadth of target activation when recall is prompted by the word "bee" alone. This single-word cue reactivates more of the context-independent associates of the target, and the consequence is that target-set-size effects are still apparent even when the related word is present in the sentence during the study phase.

Interestingly, more context-independent associates of the target words appear to be activated when related words are presented in the context of a sentence than when related words are presented contiguously with their targets in the list-learning paradigm. When the same lists of cues and targets used in the sentence experiments were studied as pairs in a list-learning experiment, target-set size failed to have a significant effect (Nelson et al., 1990). This interesting finding, however, may have been a result of the fact that the related word pairs were often separated by intervening words in the sentence experiments. This possibility was explored in Experiments $3 \mathrm{~A}$ and $3 \mathrm{~B}$.

\section{EXPERIMENTS 3A AND 3B}

The purpose of Experiments 3A and 3B was to evaluate the proposition that the temporal proximity of related words is critical to finding the target-set-size effect. Experiment $3 \mathrm{~A}$ was a list-learning experiment, and the subjects studied words varying in target-set size under one of five encoding conditions, with recall prompted by meaningfully related words. In two simultaneous conditions, the related context word and target were presented together during the study trial, for $3.0 \mathrm{sec}$ in one condition and for $1.5 \mathrm{sec}$ in the other. In two temporally asynchronous conditions, the context word either preceded or followed the target. In the context-preceding condition, the context word appeared for $1.5 \mathrm{sec}$ and terminated when the target appeared for another $1.5 \mathrm{sec}$. In the context-following condition, the reverse was true: the target appeared first for $1.5 \mathrm{sec}$, followed by the context word for another $1.5 \mathrm{sec}$. In all of these conditions, the context word also served as the test cue. Finally, in the context-absent condition, the targets were presented for $3 \mathrm{sec}$ each in the absence of any context words. This condition served as a control, with recall prompted by the same cue as was used for the other conditions.

PIER predicts that target-set-size effects will be smallest in the simultaneous conditions, intermediate in the temporally asynchronous conditions, and largest when no context words are present during the study trial. This prediction follows from the assumption that the encoding context determines the functional activation of directly related associates (Nelson, 1989; Nelson, Schreiber, \& McEvoy, 1992). Such associates are presumably automatically and implicitly activated upon reading the target and provide rapid access to related knowledge, but some of this information is likely to be contextually irrelevant (e.g., the meaning "swarming with people" is related to "hive" but only remotely related to "bee hive"). The model assumes that irrelevant meanings of the target and the related context word are rapidly inhibited when the two words are directly related (cf. Gernsbacher \& Faust, 1991). Hence, when context word and target are presented simultaneously, direct associates unrelated to the union of the two words are inhibited (e.g., given "bee hive," "honey" is presumably activated but not "people'). As a consequence, set-size effects should not be obtained, and this result has now been replicated many times (e.g., Nelson \& Friedrich, 1980; Nelson, Schreiber, \& McEvoy, 1992).

According to PIER, the functional activation of related associates produces an implicit representation during the study trial that can be searched during testing. The implicit and explicit search processes are mutually exclusive, and the implicit search process simply adds to the overall level of recall. The amount added, however, depends on the size of the functionally activated set, with more added when the set is small. Sampling within the set is assumed to be serial and self-terminating, and the smaller the number of potential competitors, the greater the chances of sampling the target as a member of the set. In the simultaneous condition, because of the inhibition of irrelevant associates, the activated set is presumed to be smaller than the potential set and essentially equivalent for words with small and large sets. In contrast, when the target is presented alone, each of its direct associates is potentially relevant and all are functionally activated. 
As a result, target-set-size effects should be apparent during testing and recall should be lower than in the simultaneous case. In the temporally asynchronous conditions, the context word is presented during study, but because it occurs before or after the target, the inhibition of irrelevant associates should not be as effective. As a consequence, target-set-size effects should be evident, but they will not be as large as when context words are unavailable.

\section{Method}

Design and Subjects. The design formed a $5 \times 2$ factorial in which encoding conditions were manipulated between subjects and target-set size was manipulated within subjects. The five conditions were (1) simultaneous-3 sec, (2) simultaneous- $1.5 \mathrm{sec}$, (3) context precedes target, (4) context follows target, and (5) the contextabsent control in which only the targets were studied. As in the previous experiments, the targets had either small or large associative set sizes. Eighteen subjects served in each between-subject condition, and they were assigned to conditions in replication blocks.

Materials and Procedure. The lists of cues and targets were identical to those used in Experiments 1 and 2. The presentation conditions were similar, except that the materials were shown on a Macintosh Plus computer and the timing conditions differed according to condition. In addition, there was a 1.5 -sec interval between each pair of related words and between each individual word in the nocontext control condition.

When the test cue was present during study, the subjects were told to focus on the relationship between the related words and that the word appearing on the left would be used later as a cue to help them recall the word appearing on the right. All subjects received the same instructions regardless of the timing manipulation. When the context word was absent during study, the subjects were told to remember as many words as possible. These subjects were essentially given an extralist cued-recall test. All subjects studied and were tested on five famous names in accordance with their condition before being presented with the experimental list. Self-paced testing instructions were used, with guessing encouraged but not required. After recalling the target aloud, the subject typed the response into the computer.

\section{Results and Discussion}

The results are shown in Table 3. Examination of the marginal means showed that the four context conditions produced approximately equal levels of recall, and that all of these conditions produced a higher level of recall than the context-absent control condition. Target-set-size effects were apparent, but these effects varied considerably and predictably across the various encoding conditions. Target-set-size effects were most apparent in the no-context condition involving extralist cued recall $(20 \%$ difference), next most apparent when the context word preceded or followed the target (average 10\% difference), and least apparent when the context word was presented simultaneously with the target (average $1 \%$ difference). The analysis of variance indicated that the effects of encoding conditions $\left[F(4,85)=23.27, M S_{\mathrm{e}}=.020, L S D=\right.$ $.07]$, target-set size $\left[F(1,85)=29.46, M S_{\mathrm{e}}=.010\right]$, and the interaction of these sources $[F(4,85)=5.28, L S D=$ .07] were all reliable sources of variance.

These findings indicate that when a directly related word is present the temporal relationship between context word and target is critical to obtaining target-set-size effects. This finding is consistent with PIER and suggests that setsize effects were not eliminated in the context-present sentence conditions of the previous experiments because the critical items were generally separated by other words to maintain sentence flow. Set-size effects are clearly eliminated when the critical words are presented simultaneously, regardless of whether the presentation rate was at 1.5 or $3.0 \mathrm{sec}$. The two presentation rates were included initially because we were unsure as to which rate would provide the most appropriate baseline for the asynchronous conditions. As the results show, it does not appear to matter because minimal differences between the two simultaneous context conditions were obtained. Furthermore, it does not appear to matter whether the context word appears slightly before or slightly after the target. This manipulation did not affect the overall level of recall, nor did it influence the magnitude of the targetset-size effect. What does appear to affect the magnitude of target-set-size effects is the presence of a directly associated context word during encoding and the timing of this context word in relation to the target. Without the context, target-set-size effects are robust, and with the context, these effects vary in magnitude with the proximity of the critical items.

The proximity effects obtained in Experiment 3A, however, may be limited to the use of word lists and may not be found in sentences. For example, Foss (1982) investigated semantic priming effects when prime-target word pairs were embedded in sentences and found that the amount of facilitation in target processing was the same whether the prime preceded the target by 12 or by 1.5 words. Proximity, however, had a large effect when the words were presented in lists rather than in sentences. There are many differences between Foss's work and the present studies, but his findings suggest that the proximity

Table 3

Probability of Correct Recall as a Function of Encoding Conditions and of Target-Set Size

\begin{tabular}{lccc}
\hline & \multicolumn{2}{c}{ Target-Set Size } & \\
\cline { 2 - 3 } Encoding Conditions & Small Target & Large Target & Mean \\
\hline Simultaneous-3.0 sec & .93 & .89 & .91 \\
Simultaneous-1.5 sec & .85 & .87 & .86 \\
Context precedes target & .90 & .80 & .85 \\
Context follows target & .91 & .81 & .86 \\
Context-absent control & .72 & .52 & .62 \\
Mean & .86 & .78 & \\
\hline
\end{tabular}


effects obtained with the word lists of Experiment 3A may not generalize to sentences. Experiment $3 \mathrm{~B}$ was designed to test this possibility. We used the target words and test cues of the previous experiments, but the sentences were rewritten to manipulate the number of words intervening between the context word and the target (see Appendix B). In the close and distant conditions, an average of .86 $(S D=.65)$ and $5.21(S D=1.24)$ words intervened, and this manipulation was crossed with target-set size. The sentences were presented for study and were tested using the same procedures as used in Experiments 1 and 2, with the context words serving as the test cues. Forty subjects participated, with 20 assigned to the close condition and 20 assigned to the distant condition. The probabilities of correct recall for targets having small and large set sizes were, respectively, .68 and .67 in the close condition and .75 and .67 in the distant condition. Planned comparisons indicated that the set-size effect was significant in the distant condition $\left[F(1,38)=5.60, M S_{e}=.015\right]$, but not in the close condition $(F<1)$.

These findings are consistent with the results of Experiment $3 \mathrm{~A}$ in suggesting that the proximity of a context word and a related target is critical for obtaining targetset-size effects. When a related word appears contiguously with the target in a list or in a sentence, target-set-size effects are not obtained. In contrast, when a related word is either missing or is present but distant from the target, targets with smaller sets of associates have an advantage in cued recall. Distance can be manipulated in a temporal-spatial sense as in Experiment 3A, or it can be introduced by presenting the words in sentences with other words intervening between the related items as in Experiment 3B. These findings indicate that the effects of related context words are determined more by proximity than by whether the targets are presented in lists or in sentences. Although the context provided by unrelated words appearing in a sentence produces some constraints on the meaning of the target, the inhibition of irrelevant associates appears to be closely controlled by the proximity of related words. When related words are contiguous or nearly contiguous, related but irrelevant associates are inhibited; when such words are absent or distant, irrelevant associates are activated and can be used to support later recall if the test cue engenders a search of implicit memories.

The presence of proximity effects in our sentence experiment and the absence of such effects in Foss's (1982) studies may have been due to differences in tasks, procedures, or materials. More interestingly, the discrepancy may be related to the information constrained by the sentence context and by a pair of related words. The sentential context may constrain the kind of information that is activated about a word but not the amount of information. The sentential context may activate new information not activated when a word is experienced out of context (Barsalou, 1982), while at the same time the context inhibits irrelevant information linked to specific words appearing in the sentence (e.g., Gernsbacher et al., 1990;
Seidenberg et al., 1982). The result is that the total amount of information may remain relatively constant. The kind-amount distinction is speculative, but it explains the persistence of set-size effects in sentence contexts without our having to assume that sentential context does not constrain meaning.

\section{GENERAL DISCUSSION}

The purpose of these experiments was to determine whether the number of associates linked to a word presented in the context of a sentence would affect its recall. Target-set-size effects reflect an influence of the number of associates linked to a word, and, as shown by both present and past findings, these effects are robust. Cued recall consistently varies with associative set size under a variety of conditions (e.g., Nelson \& Friedrich, 1980). Nevertheless, set-size effects are reduced when the targets are studied in the presence of meaningfully related context words, and this reduction provided a reason to believe that such effects might not be obtained when the items are presented in sentences. Even in simple sentences such as "Tom held the baby," the semantic constraints created in the process of comprehending the sentence may have been sufficient to eliminate target-set-size effects.

Despite this possibility, target-set-size effects were obtained in the simple sentences and in sentences in which the meaningfully related word serving as the test cue was also present during study. Set-size effects were found in sentences under conditions that normally favor the elimination of such effects in list-learning experiments. The failure to eliminate the effect when the related context word was available during study was a result of the separation of the critical words in the sentence. The results of Experiment 3A and those of the follow-up study indicate that the magnitude of the set-size effect depends on the proximity of the target to the related context word. Contiguous presentation of context word and target in either a list or a sentence essentially eliminates the effect, whereas temporal separations of $1.5 \mathrm{sec}$ or the presence of a few intervening words appear to be sufficient to produce the effect. In general, these results and other findings derived from list-learning experiments support the conclusion that target-set-size effects will be obtained in cued recall whenever the context fails to provide effective semantic or associative constraints. Set-size effects occur when words are studied in the absence of modifying context words, in the presence of rhyming or unrelated words, or, when separated, in the presence of related words.

Although target-set-size effects may not be found in other types of sentences or in larger units of discourse, the appearance of such effects in sentence material can be used to indicate when the meaning of critical words in the sentence has not been fully constrained by the context. Comprehension presumably involves the construction of a representation that can be used to support recall, answer questions, and so forth, and subjects bring 
a wealth of prior associative knowledge to such tasks, only some of which may be relevant. The theoretical problem is to control the construction process so that ambiguities produced by irrelevant knowledge can be avoided. Topdown models control irrelevant knowledge by assuming that scripts, frames, or schemata constrain the process beforehand by creating expectancies that allow the comprehender to predict what is relevant (e.g., Schank \& Abelson, 1977). However, attempts to create such expectancies would have been ineffective for the present materials because the sentences were not related by a common theme. In contrast to top-down models, Kintsch's (1988) construction-integration model controls irrelevant knowledge by assuming processes that inhibit such knowledge after it has been accessed. Each concept in the sentence accesses its closest associates regardless of the supporting context, and a cyclical integration process is then used to exclude irrelevant associates.

The presence of target-set-size effects in sentence contexts provides support for the assumption that the closest associates of a word are activated and can play a role in the retrieval of sentence material (Kintsch, 1988). Although subjects explicitly focus their processing resources on the presented words in order to meet task demands (e.g., Just \& Carpenter, 1980), preexisting knowledge in the form of close associates is implicitly activated in the process of constructing a meaning for the sentence. The representation of discourse appears to include both explicitly encoded information (e.g., the linkages created among the words "Tom-held-baby") and implicitly activated information related to the associates of each element (e.g., "Tom" = person, male, and so forth, "held" = grasp, carry, and so forth, and "baby" = child, cry, and so forth). In Kintsch's constructionintegration model, such knowledge is activated in the construction phase with information irrelevant to the sentence inhibited in the integration phase (e.g., after encoding the above sentence, associates of "baby" such as small, cute may be inhibited). The present findings are consistent with this model and indicate that the constraints provided by presenting associatively related words in the same sentence are likely to be highly sensitive to the relative placement of the words. The intervention of just a few words between the critical items may be sufficient to prevent the inhibition of irrelevant information or at least to reduce the effectiveness of this process.

The construction-integration model was designed to explain discourse comprehension processes, and PIER was designed to explain set-size effects in various memory tasks. Although devised for different purposes, both models incorporate a role for preexisting associative knowledge. PIER assumes that the encoding of a list of familiar words involves the creation of dual representations, one produced as a result of explicit processing operations and one produced as a result of the implicit activation of related associates. In the cued-recall task, both representations make independent contributions to recall. Some targets are recalled as a result of sampling information created as a result of explicit processing activities such as sentence rehearsal (e.g., Raaijmakers \& Shiffrin, 1981 ), and some are recovered as a result of sampling information implicitly activated during the study trial and reactivated during testing by the cue (Nelson, 1989). Target-set-size effects are produced as a result of sampling associates of the target reactivated by the test cue during testing. When fewer associates have been functionally activated during study because of the nature of the encoding context, words having smaller associative set sizes no longer have a relative advantage in recall. Similarly, the relative advantage is eliminated when the test cue constrains the target to the point that implicit search processes are not engaged. In the present context, the important point is that information implicitly activated during encoding can provide an alternative means for retrieving the target during testing and such access occurs regardless of whether the target has been studied as one word in a list of words or as an important constituent in a sentence. The critical determinant is not whether the word has been encoded in a list or in a sentence but whether meaningfully related words are present in close proximity and whether the test cue promotes or restricts the breadth of the implicit search process.

\section{REFERENCES}

Bahrick, H.P. (1970). Two-phase model for prompted recall. Psychological Review, 77, 215-222.

BArSALOU, L. W. (1982). Context-independent and context-dependent information in concepts. Memory \& Cognition, 10, 82-93.

Foss, D. J. (1982). A discourse on semantic priming. Cognitive Psychology, 14, 590-607.

Gernsbacher, M. A., FAUST, M. E. (1991). The mechanism of suppression: A component of general comprehension skill. Journal of Experimental Psychology: Learning, Memory, \& Cognition, 17, 245-262.

Gernsbacher, M. A., Varner, K. R., Faust, M. E. (1990). Investigating differences in general comprehension skill. Joumal of Experimental Psychology: Leaming, Memory, \& Cognition, 16, 430-445.

Just, M. A., CARPENTER, P. A. (1980). A theory of reading: From eye fixation to comprehension. Psychological Review, 87, 329-354.

KINTSCH, W. (1988). The role of knowledge in discourse comprehension: A construction-integration model. Psychological Review, 95, 163-182.

NELSON, D. L. (1989). Implicitly activated knowledge and memory. In C. Izawa (Ed.), Current issues in cognitive processes: The Tulane Floweree Symposium on Cognition (pp.369-387). Hillsdale, NJ: Erlbaum.

Nelson, D. L., Bajo, M. T., Casanueva, D. (1985). Prior knowledge and memory: The influence of natural category size as a function of intention and distraction. Joumal of Experimental Psychology: Learning, Memory, \& Cognition, 11, 94-105.

NeIson, D. L., CANAS, J., BAJo, M. T. (1987). The effects of natural category size on memory for episodic encodings. Memory \& Cognition, 15, 133-140.

Nelson, D. L., Friedrich, M. A. (1980). Encoding and cuing sounds and senses. Journal of Experimental Psychology: Human Learning \& Memory, 6, 717-731

Nelson, D. L., Keelean, P. D., \& Negrao, M. (1989). Word-fragment cuing: The lexical search hypothesis. Journal of Experimental Psychology: Learning, Memory, \& Cognition, 15, 388-397.

Nelson, D. L., \& McEvoy, C. L. (1979). Encoding context and set size. Joumal of Experimental Psychology: Human Learning \& Memory, 5, 292-314.

Nelson, D. L., McEvoy, C. L., Janczura, G. A., \& XU, J. (1992). Reductions in the influence of implicitly activated memories on recall: Decay, interference, or inhibition? Manuscript submitted for publication. 
Nelson, D. L., McEvoy, C. L., \& Schreiber, T. A. (1990). Encoding context and retrieval conditions as determinants of the effects of natural category size. Journal of Experimental Psychology: Learning, Memory, \& Cognition, 16, 31-41.

Nelson, D. L., \& SCHREIBER, T. A. (1992). Word concreteness and word structure as independent determinants of recall. Joumal of Memory and Language, 31, 237-260.

Nelson, D. L., Schreiber, T. A., McEvoy, C. L. (1992). Processing implicit and explicit representations. Psychological Review, 99, 322-348.

ONIFER, W., SWINNEY, D. A. (1981). Accessing lexical ambiguities during sentence comprehension: Effects of frequency of meaning and contextual bias. Memory \& Cognition, 9, 225-236.
RaAijmakers, J. G. W., \& Shiffrin, A. M. (1981). Search of associative memory. Psychological Review, 88, 93-134.

Schank, R. C., A ABELSON, R. P. (1977). Scripts, plans, goals, and understanding. Hillsdale, NJ: Erlbaum.

Seidenberg, M. S., Tanenhaus, M. K., Leiman, J. M., BienKowskı, M. (1982). Cognitive Psychology, 14, 489-537.

TABOssI, P. (1988a). Accessing lexical ambiguity in different types of sentential contexts. Journal of Memory \& Language, 27, 324-340.

TABossI, P. (1988b). Effects of context on the immediate interpretation of ambiguous nouns. Journal of Experimental Psychology: Learning, Memory, \& Cognition, 14, 153-162.

UNDERWOOD, B. J., \&CHULZ, R. W. (1960). Meaningfulness and verbal learning. New York: Lippincott

\section{APPENDIX A}

\section{Materials for List 1 of Experiments 1 and 2}

\section{Context Word Absent:}

John watched the hive. Cathy heard and began to laugh.

Jim feared the dark

Mary worked with her needle.

Henry yelled at the cat.

Susan knew it was round.

Bob wanted to stop.

Bill met the wife.

Martha received an exam.

Molly waited for the pony.

Larry noted the pond.

Jane kept her sight.

Tom held the baby.

Jill asked about the bus.

Sam understood about the dirt.

Joe put on a robe.

Lynn avoided the door.

Sally enjoyed the fire.

Frank found the moose.

Laura expected the rain.

Louis touched the silk.

Eileen loved the flower.

George cut the rope.

Kim wished she would think.

\section{Context Word Present:}

John watched the bee fly around the hive. Cathy heard the joke and began to laugh. Jim feared the night because it was dark. Mary worked with her thimble and needle. Henry saved the mouse and yelled at the cat. Susan knew a circle was round.

Bob saw the sign and wanted to stop. Bill met the spouse and then his wife. Martha needed a test and received an exam. Molly waited for the horse and pony. Larry noted that the lake became a pond. Jane was older but kept her eye sight.

Tom held the child who was yesterday's baby. Jill wanted the truck and asked about the bus.

Sam understood about the mud and dirt.

Joe put on slippers and a robe.

Lynn climbed in the window and avoided the door.

Sally lit a $\log$ and enjoyed the fire.

Frank found the elk with the moose.

Laura expected the storm and its rain.

Louis touched the satin and the silk.

Eileen loved the plant and its flower.

George cut the string and then the rope.

Kim wished her brain would think.

\section{Materials for List 2 of Experiments 1 and 2}

\section{Context Word Absent:}

Tom held the calf. Jill asked about the clock. Sam understood his job.

Kim wished it would hop.

Laura expected to be sad.

Frank found the razor.

Sally enjoyed the king.

Lynn avoided the sand.

Louis touched the glue.

Joe put on a sail.

George cut the twig.

Eileen loved the bride.

\section{Context Word Present:}

Tom stood near the cow and held the calf. Jill needed the time and asked about the clock. Sam accepted the task and understood his job. Kim wished it would jump and hop. Laura expected grief and was sad. Frank found the blade before the razor. Sally enjoyed the prince and the king. Lynn avoided the beach because of the sand. Louise touched the sticky glue.

Joe moved the boat and put on a sail. George cut from branch to twig. Eileen loved the wedding and the bride. 
Susan knew teachers to admire.

John watched the wine.

Henry yelled for the cheese.

Bob wanted to elect.

Cathy heard the gun.

Jim feared the money.

Bill saw the neck.

Jane kept it soft.

Mary worked with her pin.

Molly waited for the melt.

Martha received a test.

Larry noted the crunch.
Susan knew teachers to like and admire.

John watched the flask as he poured the wine.

Henry yelled at the mouse that ate the cheese.

Bob wanted to cast his vote and elect.

Cathy heard about the shoot and brought her gun.

Jim feared the bribe but took the money.

Bill saw the throat and then the neck.

Jane kept her pillow soft.

Mary worked with her needle and pin.

Molly waited for the butter to thaw and melt.

Martha needed to evaluate a test.

Larry noted the cereal was crisp and went crunch.

Note-The targets (last word in each sentence) in the first 12 sentences in each context condition had small associative sets, and those in the last 12 sentences had large associative sets. Although no words were underlined during presentation, the underlined words in the context condition served as test cues for the targets in both conditions.

\section{APPENDIX B}

\section{Materials for List 1 in Experiment 3B}

\section{Close Sentences:}

John resigned himself and watched the bee hive.

Cathy heard the long and involved joke and had to laugh.

Jim was afraid of the noise and because the long night was dark.

Mary sewed in her chair with her thimble and needle.

Henry was exceptionally pleased when he saved the mouse cat.

Susan pedalled too fast and had to slow up to make the circle round.

Bob was speeding in his car before he saw the sign to stop. Bill looked casually around the room before he saw his spouse his wife.

Martha wanted practice and requested a short test before the exam.

Molly waited patiently with her daughter for the horse and pony. Larry noted the biologist's predicted change from lake to pond. Jane had surgery for hours but did not lose her eye sight. Tom played with and then held with pleasure the child yet baby. Jill asked politely and then transfered from truck to bus.

Sam understood after the long explanation how mud becomes dirt.

Joe relaxed after the long day and put on his slippers and robe. Dave painted around the large ornate window and door.

Sally lit the paper and enjoyed sitting in front of the log fire. Frank found the pasture where there were both elk and moose. Maura expected and looked forward to the storm and rain. Louis bought cloth for his store and found both satin and silk. Eileen immediately returned to her home to plant her flower. George wound the long thick string into a rope.

Kim wished she was not so tired and that her brain would think.
Distant Sentences:

John watched the bee fly around the hive.

Cathy heard the joke thought a moment and had to laugh. Jim was afraid of the night because it was dark.

Mary sewed with her thimble on her finger using her needle. Henry was pleased when he saved the mouse and yelled at the cat.

Susan pedalled the circle too fast then slowed as she went round.

Bob was speeding and saw the sign and tried hard to stop. Bill looked around and saw the spouse and then he saw the wife.

Martha wanted a short test prior to taking the full exam.

Molly waited for the horse and was finally given a pony. Larry noted that the lake over the years had become a pond. Jane had surgery on her eye and did not lose her sight.

Tom played with and held the child who really was still a baby. Jill transfered from the truck and asked politely about the bus. Sam understood mud and that without water it becomes dirt.

Joe relaxed and put on slippers but could not find his robe. Dave painted the window then hung the large ornate door.

Sally lit the paper under the log and enjoyed the fire.

Frank found the elk in the pasture with the moose.

Maura expected the storm and looked forward to its rain. Louis bought the satin before he was able to find the silk. Eileen immediately retumed to plant the tall water-loving flower. George wound the thick string and then the long rope.

Kim wished her brain was not so tired and would think.

\section{Materials for List 2 of Experiment 3B}

\section{Close Sentences:}

Tom stood with pride near his cow and calf.

Jill needed the work and was glad to punch the time clock. Sam accepted and understood the difficult task as his job. Kim wished that the rabbit near the fence would jump and hop. Maura expected loss and because she anticipated grief was sad.

\section{Distance Sentences:}

Tom stood near the cow and held the young calf. Jill needed the time and asked about the clock. Sam accepted the task and with instructions understood his job. Kim wanted the rabbit to jump the fence but all he did was hop. Maura expected grief but despite her anticipation she was sad. 
Frank searched for a long time before locating the blade and razor.

Sally enjoyed talking to the tall handsome prince soon king.

Lynn avoided going on windy days because of the beach sand. Louise accidentally touched the bottle of sticky glue.

Joe strained his back while he was raising the boat sail.

George cut and trimmed the bushy oak tree from branch to twig.

Eileen loved all of the festivities and the wedding bride.

Susan knew many teachers that she could like and admire.

John resigned himself as he carefully lifted the old flask of wine.

Henry felt sorry for the animal and left the mouse some cheese.

Bob understood his lofty position and that to vote was to elect.

Cathy heard of the contest and could not wait to shoot her gun.

Jim was fearful but willing so he accepted the bribe money.

Bill detected the signs when he saw the throat and neck.

Jane injured her back so she liked her pillow soft.

Mary sewed the work in her chair for hours using needle and pin.

Molly waited patiently to see the stick of butter thaw and melt.

Martha asked for the scoring procedure used to evaluate the test. Larry ate the sugary cereal and was pleased by its crisp crunch.
Frank found the blade and after a long search he located the razor.

Sally enjoyed talking to the prince who intended to rule after the king.

Lynn avoided the beach because of the sand.

Louise touched the sticky part of the bottle used for glue. Joe moved the boat in the water and raised its sail.

George cut the branch and trimmed the tree to its last twig. Eileen loved the wedding all the festivities and the bride. Susan knew teachers to like and others that she could admire. John watched the flask as he poured the red wine.

Henry yelled at the mouse that ate the swiss cheese.

Bob understood his position and that to vote in this case was to elect.

Cathy heard about the turkey shoot and brought her father's gun. Jim feared the bribe but greedily took the easy money.

Bill saw the signs in the throat and then looked at the neck. Jane liked her pillow to be clean and very soft.

Mary sewed with a needle and to keep the work together she used a pin.

Molly waited for the ice to thaw because she wanted to see it melt.

Martha asked to evaluate the scoring procedure for the test. Larry noted the cereal was crisp and when chewed it had a good crunch. 